\title{
What happens to the pylorus after pyloromyotomy?
}

\author{
N M OKORIE, ${ }^{*}$ J A S DICKSON, ${ }^{*}$ R A CARVER, $\dagger$ AND G M STEINER $\dagger$ \\ ${ }^{*}$ Sub-department of Paediatric Surgery, and †Department of Radiology, Children's Hospital, Sheffield
}

SUMMARY The rate of return to normal of the pylorus muscle in infantile hypertrophic pyloric stenosis was measured by ultrasound, using a simple pyloric volume calculation and a pyloric muscle index. The muscle hypertrophy took from two to 12 weeks to resolve.

First performed in $1911,{ }^{1}$ Ramstedt's pyloromyotomy has long been the standard treatment for infantile hypertrophic pyloric stenosis. Its success has left knowledge of what happens to the hypertrophied pyloric muscle dependent on measurements taken after the increasingly rare deaths and occasional later laparotomies. ${ }^{2}$ These have shown that the pylorus eventually returns to normal leaving only a hairline longitudinal scar, and when combined with an earlier ultrasound study ${ }^{13}$ have suggested that the pyloric muscle returns to normal within two to six weeks of the operation.

Ultrasound identification of pyloric hypertrophy started in $1977 .{ }^{4}$ The basic technique is simple but the interpretation of the results has been complicated by the overlap of the upper limit of normal and the lower limit accepted as indicating muscle hypertrophy (fig 1). ${ }^{5}$ This overlap also affects the interpretation of postoperative changes.

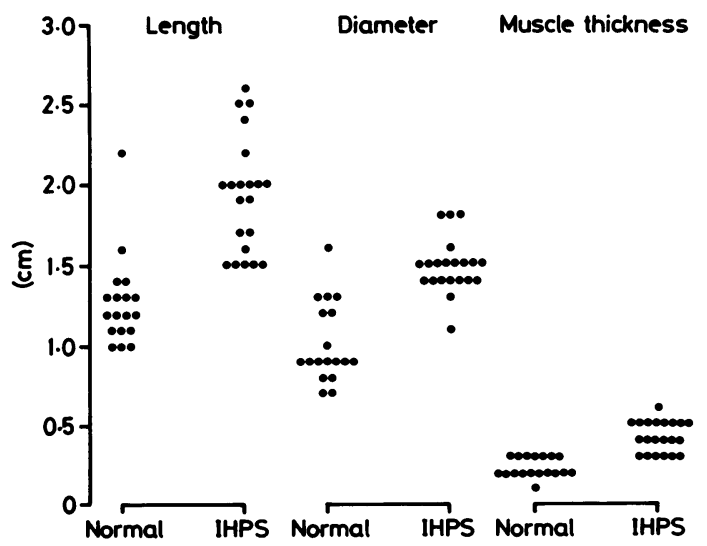

Fig 1 Measurements of the pylorus in infantile hypertrophic pyloric stenosis (IHPS) and in normal controls.

\section{Patients and methods}

Twenty five babies with a diagnosis of infantile hypertrophic pyloric stenosis confirmed at operation and 24 controls were studied. The protocol was approved by the local ethical committee and informed consent obtained for each child. The only reason for exclusion of a child from the study was a home too far from hospital to return easily for the additional ultrasound scans.

Initially the pyloric muscle was studied preoperatively and at two, four, and six weeks postoperatively. It was soon obvious that this was not a long enough follow up period and further measurements were taken at eight, 10, 12, and 14 weeks. Finally, follow up studies were restricted to before, and six and 12 weeks after the operation.

Criteria for the ultrasound diagnosis of infantile hypertrophic pyloric stenosis had first to be established. ${ }^{5}$ The pylorus was assumed to be a hollow cylinder. Its muscle volume was calculated from the formula $\pi T L(D-T)$ where $T$ is the thickness, $L$ the length, and $D$ the diameter of the pylorus in $\mathrm{cm}$. The accuracy of the ultrasound measurements was checked in the operating theatre for affected babies. This volume showed an overlap between cases and controls (normal range, 0.26-1.96 cm babies with infantile hypertrophic pyloric stenosis, $1.50-4.90 \mathrm{~cm}^{3}$ ). When the volume was divided by the weight of the baby in $\mathrm{kg}$, a muscle index was obtained, which gave a complete statistical separation (normal range, $0 \cdot 08-0 \cdot 28$; babies with infantile hypertrophic pyloric stenosis, 0.46-1.26) (fig 2). Weight loss increases the pyloric muscle index and one very marasmic infant without infantile hypertrophic pyloric stenosis measured at a postmortem examination had a value in the range for the affected babies. In a series of five cases and four controls, the validity of correcting for baby size was checked by dividing the volume by the baby's surface area $\left(\mathrm{m}^{2}\right)$, 


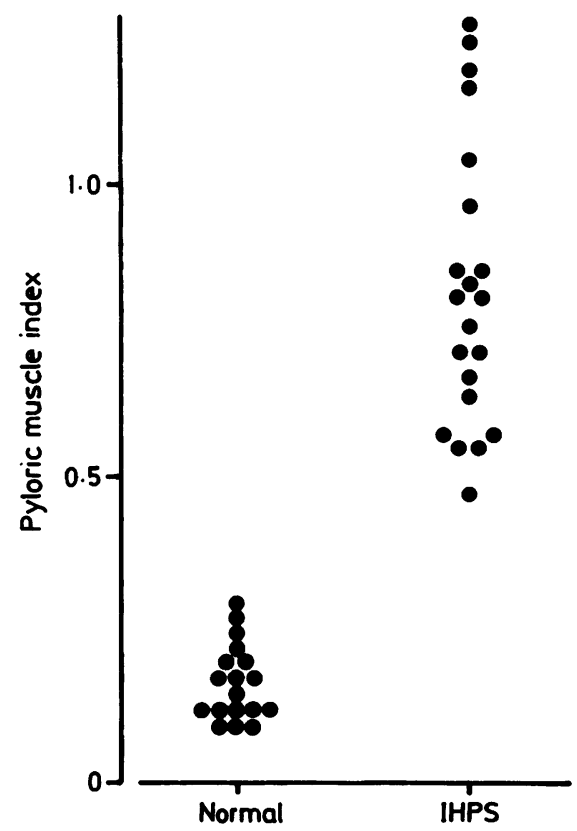

Fig 2 Pyloric muscle index in infantile hypertrophic pyloric stenosis (IHPS) and in normal controls. Pyloric muscle index = pyloric muscle volume divided by body weight $(\mathrm{kg})$.

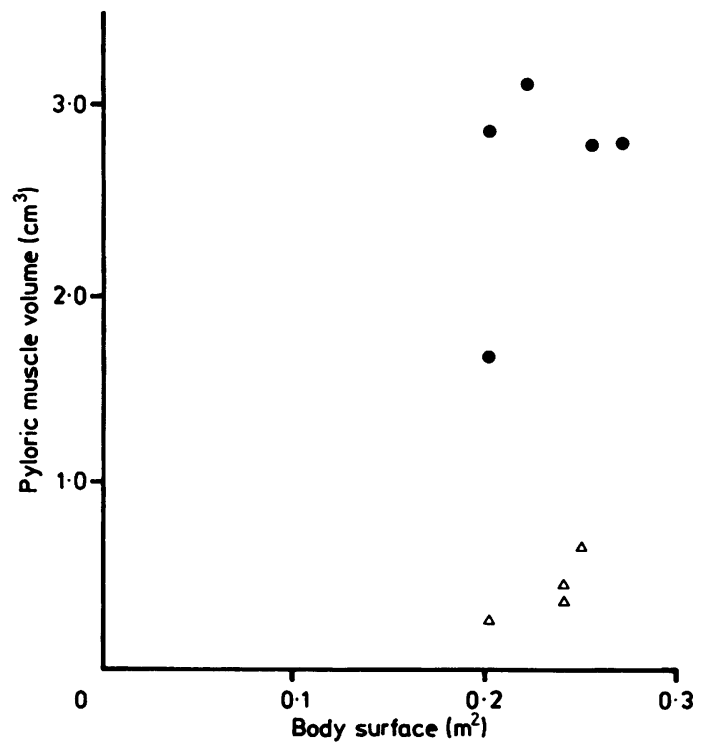

Fig 3 Pyloric muscle volume divided by body surface infantile hypertrophic pyloric stenosis (O) and normal controls $(\triangle)$. which was obtained from the nomogram derived from the formula of Du Bois and Du Bois (fig 3). ${ }^{6}$ This also gave a good separation, confirming the acceptability of the method and that failure to thrive was not a major feature of infantile hypertrophic pyloric stenosis in our series.

\section{Results}

The time to return to a normal pyloric muscle volume and to a normal pyloric index was measured using the control values as a normal base line. The pyloric muscle volume returned to normal in six weeks in six babies, but took 12 weeks in three of the last nine patients. If the pylorus is growing with the baby it will not return to the preoperative size. We have therefore used the muscle index, which takes this into account, to assess progress. Fig 4

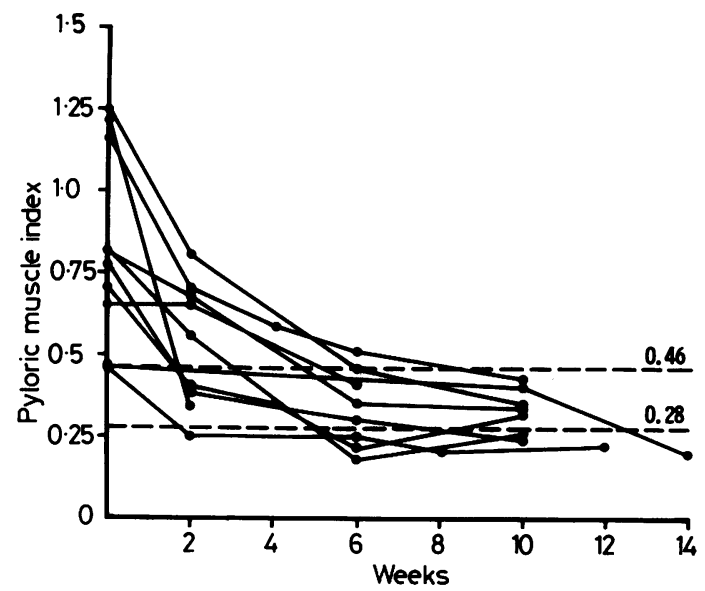

Fig 4 Changes in pyloric muscle index after pyloromyotomy.

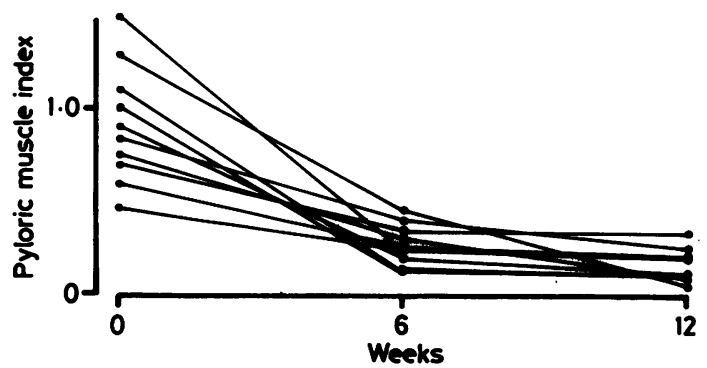

Fig 5 Pyloric muscle index preoperatively, and at six and 12 weeks postoperatively. 
Table Changes in pyloric muscle index after pyloromyotomy

\begin{tabular}{|c|c|c|c|c|c|c|c|c|}
\hline & \multirow{2}{*}{$\begin{array}{l}\text { Day of } \\
\text { operation }\end{array}$} & \multicolumn{7}{|c|}{ Time postoperative (weeks) } \\
\hline & & 2 & 4 & 6 & 8 & 10 & 12 & 14 \\
\hline $\begin{array}{l}\text { No of patients } \\
\text { No }(\%) \text { of patients with index: }\end{array}$ & 25 & 19 & 11 & 17 & 3 & 12 & 8 & 6 \\
\hline $\begin{array}{l}\leqslant 0.46^{*} \\
\leqslant 0.28 \dagger\end{array}$ & $\begin{array}{l}\mathbf{0} \\
\mathbf{0}\end{array}$ & $\begin{array}{r}10(53) \\
4(21)\end{array}$ & $\begin{array}{l}8(73) \\
4(36)\end{array}$ & $\begin{array}{r}15(88) \\
8(47)\end{array}$ & $\begin{array}{l}3(100) \\
3(100)\end{array}$ & $\begin{aligned} 12(100) \\
7(58)\end{aligned}$ & $\begin{array}{l}8(100) \\
8(100)\end{array}$ & $\begin{array}{l}6(100) \\
6(100)\end{array}$ \\
\hline
\end{tabular}

*Diagnostic of pyloric stenosis; †normal (non-pyloric stenosis).

shows the pattern for 10 of the first 20 patients and fig 5 the simplified pattern, taking measurements preoperatively, and at six and 12 weeks. The table records the progress of all patients showing that the index falls below the lower limit for the diagnosis of infantile hypertrophic pyloric stenosis by 8 weeks, but takes 12 weeks to fall to the normal range.

\section{Discussion}

The pattern suggests that two processes are occurring simultaneously: the muscle bulk is reducing and the child is growing. The hypertrophy regresses at a variable rate but a normal size is consistently reached by 12 weeks postoperatively.

We wish to thank Mrs Jones, research secretary, for typing the paper and Mrs A Allen, senior I radiographer, for carrying out the ultrasound scans.

\section{References}

${ }^{1}$ Ramstedt K. Zur Operation der angeborenen Pylorus-stenose. Medizinische Klinik Nr 1912;42:1702-5.

2 Wollstein M. Healing of hypertrophic pyloric stenosis after the Fredet-Ramstedt operation. Am J Dis Child 1922;23:511-7.

${ }^{3}$ Sauerbrei EE, Paloschi CGB. The ultrasonic features of hypertrophic pyloric stenosis, with emphasis on the postoperative appearance. Radiology 1983;147:503-6.

4 Teele RL, Smith EH. Ultrasound in the diagnosis of idiopathic hypertrophic pyloric stenosis. N Engl J Med 1977;296:1149-50.

5 Carver RA, Okorie NM, Steiner GM, Dickson JAS. Infantile hypertrophic pyloric stenosis-diagnosis from the pyloric muscle index. Clin Radiol 1987;138:625-7.

6 Du Bois D, Du Bois E. A formula to estimate the approximate surface area if height and weight be known. Arch Intern Med 1916;17:863.

Correspondence to Mr JAS Dickson, Sub-department of Paediatric Surgery, Children's Hospital, Western Bank, Sheffield S10 2TH.

Accepted 6 May 1988 